\title{
A New Form of $\mathrm{MgTa}_{2} \mathrm{O}_{6}$ obtained by the Molten Salt Method
}

\author{
S.Nangia *, M. Thirumal *, A.K. Ganguli ${ }^{*}$ and P.L. Gai \# \\ *Department of Chemistry, Indian Institute of Technology, New Delhi 110016, India \\ \# DuPont, Central Research and Development, Wilmington DE 19880-0356 and University of \\ Delaware, Newark, Delaware, USA.
}

$\mathrm{MgTa}_{2} \mathrm{O}_{6}$ is an important dielectric material and has uses in the microwave frequency range. $\mathrm{MgTa}_{2} \mathrm{O}_{6}$ is reported to crystallize in the trirutile structure (tetragonal, space group $\mathrm{P} 4_{2} / \mathrm{mmm}$ ) with lattice parameters of $\mathrm{a}=4.695$ and $\mathrm{c}=9.147 \AA$. Here the structure is made up of strings of edge-shared octahedra extending along the $\mathrm{c}$ - direction and these strings are linked together by sharing corners. The edge-sharing occurs at opposite edges in each octahedron leading to linear octahedral strings in the trirutile structure. The above structure may be obtained by solid state reactions of $\mathrm{MgCO}_{3}$ and $\mathrm{Ta}_{2} \mathrm{O}_{5}$ at high temperatures of $1200{ }^{\circ} \mathrm{C}$ to $1400{ }^{\circ} \mathrm{C}$. We have been interested to prepare oxides by alternate low temperature routes such as by the molten salt or flux method $[1,2]$. In this report we discuss our studies on the synthesis of a new form of magnesium tantalate by using a $\mathrm{NaCl}-\mathrm{KCl}$ flux.

Our starting reagents are $\mathrm{Mg}\left(\mathrm{NO}_{3}\right)_{2} \cdot 6 \mathrm{H}_{2} \mathrm{O}$ and $\mathrm{Ta}_{2} \mathrm{O}_{5}$ to which we add a $1: 1$ mixture of $\mathrm{NaCl}$ $\mathrm{KCl}$ flux. After proper mixing and grinding the mixture is heated in a furnace at $900 \mathrm{C}$ for $6 \mathrm{~h}$. the product is washed with distilled water several times and checked for absence of $\mathrm{Cl}^{-}$ions in the filtrate. This is to make sure that all the flux is washed off. The powder is then dried at 120C.

Powder x-ray diffraction studies suggest an altogether different phase as compared to the reported $\mathrm{MgTa}_{2} \mathrm{O}_{6}$, and peak overlap is also possible. To find out the probable lattice parameters we have carried out electron microscopic investigations using a combination of HRTEM and electron diffraction procedures to determine the lattice parameters. These complex multicrystalline powder compounds were found to be very beam sensitive and low electron dose conditions were therefore employed $[3,4]$. Using electron accelerating voltages of $200 \mathrm{kV}$ in a FEI CM30 HRTEM, we have succeeded in high resolution imaging and electron diffraction measurements of the compound. Crystals were tilted to different zone axes, including [12-1] projections. High precision composition analysis was carried out simultaneously using energy dispersive X-ray spectroscopy (EDX) in the HRTEM. Initial electron diffraction data have shown a dominant orthorhombic phase with approximate lattice parameters of $\mathrm{a} \sim 15.4 \AA$, $\mathrm{b} \sim 13.4$ $\AA$, and $\mathrm{c} \sim 12.2 \AA$,. Electron diffraction pattern, for example, in [12-1] and the corresponding lattice image are shown in FIG.1 and FIG. 2, respectively. EDX composition analysis of the compound is shown in FIG.3. Some minor phases are also present [FIG.4]. We have satisfactorily indexed the powder $x$-ray diffraction pattern on the basis of the above orthorhombic cell and all the lines could be indexed. The refined lattice parameters of the new structure obtained are, $a=15.35(1) \AA, b=13.33(1) \AA$, and $c=12.057(9) \AA$.

References:

[1] M.Thirumal, P. Jain and A.K.Ganguli, Mat. Chem \& Phys. 70 (2001) 7.

[2] A.K. Ganguli, V. Grover and M. Thirumal, Mat. Res. Bull. 36 (2001) 1967.

[3] P.L. Gai et al., J.Phys.Chem. 96 (1992) 8206.

[4] P.L. Gai et al., Science 267 (1995) 661. 


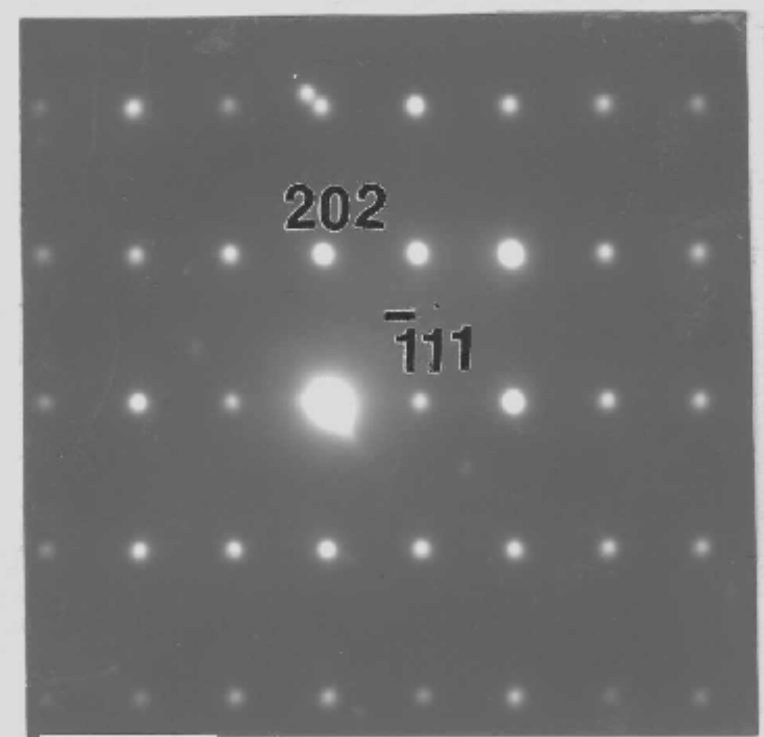

FIG. 1

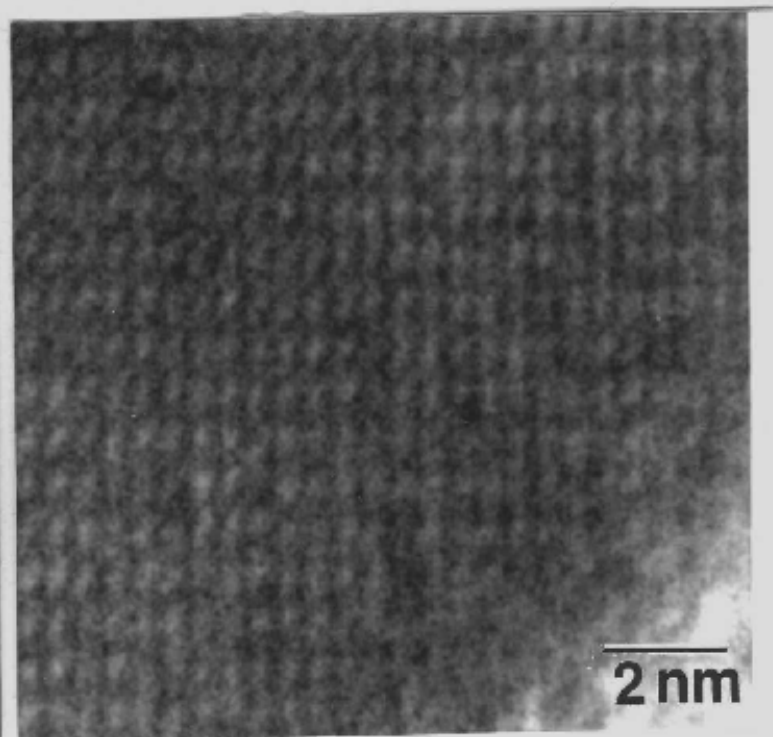

FIG.2

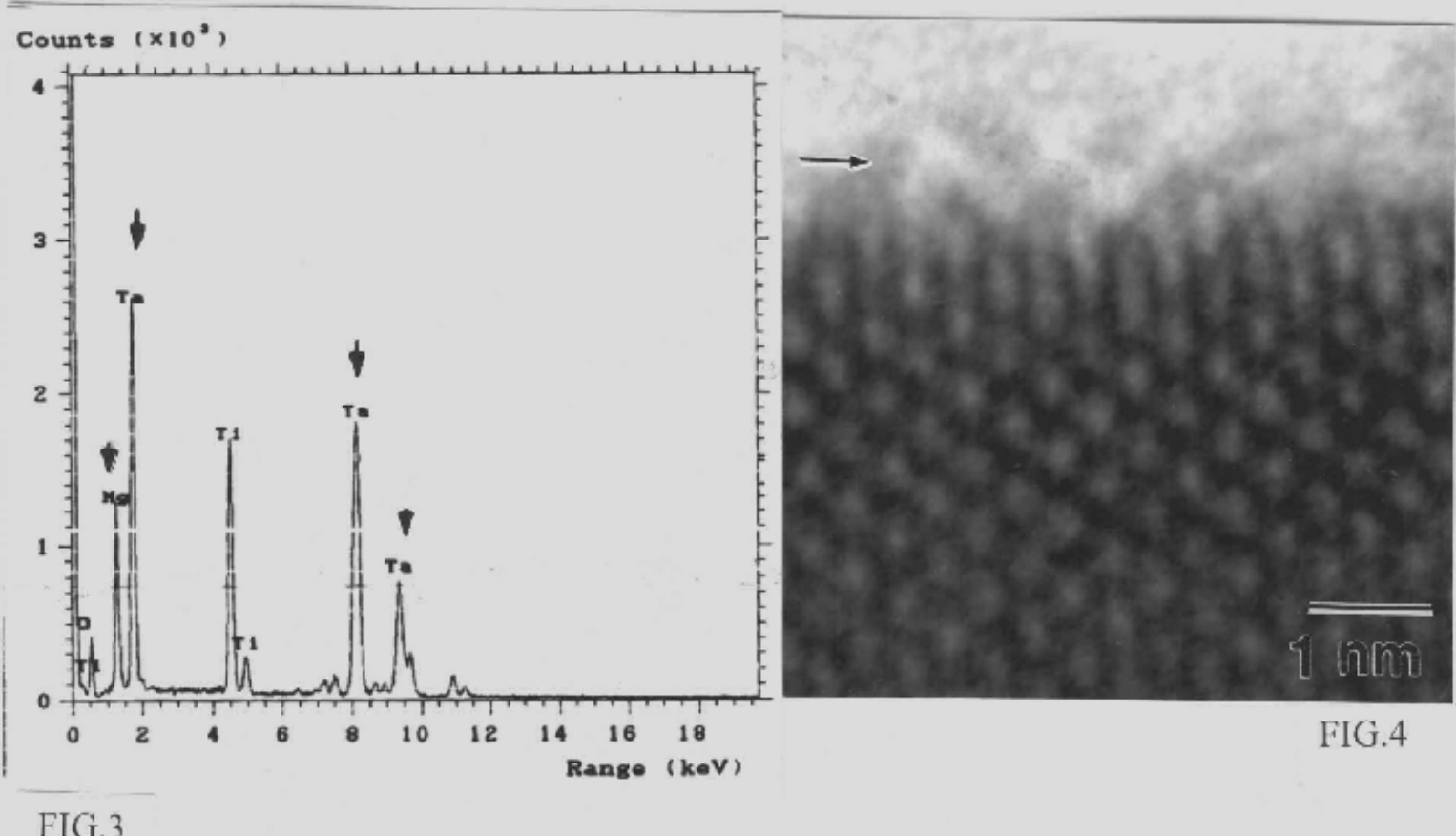

FIG. 1 Electron diffraction pattern of complex $\mathrm{MgTa}_{2} \mathrm{O} 6$ along [12-1] zone axis FIG. 2 HRTEM of the complex, beam sensitive oxide with well ordered crystals. FIG.3 X-ray composition spectrum of the oxide supported on titanium grid. FIG.4 Secondary phase observed in the $\mathrm{Mg}-\mathrm{Ta}-\mathrm{O}$ system, with surface layers. 